\title{
Modal Analysis of Femur Bone to Find out the Modal Frequencies of Different Bone Implant Materials
}

\author{
Vaishali Chaudhry, Anubhav Kumar, Shilpa N, Shwetank Avikal, Nithin Kumar K.C.
}

\begin{abstract}
Bio-mechanics is most difficult to carry out on the bone due to the modeling difficulty and complex forces acting on the bones. In this study, we consider human femur bone for modeling analysis. The modal analysis is also important as that of static analysis. We can predict the place at which the fracture occurs. The modal analysis for three different materials is carried out to find the feasible material for bone implants. These materials are Natural bone, AZ31, and Stainless steel 316L. The daily activity such as walking is used as a boundary condition in our study. The femur head is fixed and $750 \mathrm{~N}$ load is applied at the Knee joint. The results are obtained for these materials. The modal frequencies for Natural Femur bone vary from $0.328 \mathrm{~Hz}$, to $2.258 \mathrm{~Hz}$ for Mode1 to Mode 10. The modal frequencies for AZ31 vary from $1.502 \mathrm{~Hz}$ to $10.292 \mathrm{~Hz}$ for Model to Mode 10. The modal frequencies for $316 \mathrm{~L}$ vary from $3.120 \mathrm{~Hz}$ to $21.150 \mathrm{~Hz}$ for Mode1 to Mode 10. These frequencies are minimal as compared to the natural frequency of the Femur bone. AZ31 is best suited for the fabrication of bone implants because of its lightweight in comparison with $316 \mathrm{~L}$ material. Also, this is biodegradable in the human body over the period.
\end{abstract}

Keywords: Femur bone, Modal analysis, fracture, different materials, CT-Scan

\section{INTRODUCTION}

The biomechanical study deals with the understanding of the mechanical behavior of biological objects such as bones [1]. The recent development in the field of computational made the analysis of complicated systems easier and less time-consuming [2]. In the human body, the femur bone is the most important and longest, it is important because it supports the body and the whole body weight is transferred to the leg [3]. So we required to understand the behavior of this bone and at the same time, we required to find the feasible implant materials through Finite element analysis [4]. Femur bone fractures generally occur due to the trauma caused by accidents [5]. The modal analysis is one of the criteria to analyze the frequencies for different materials such that we can predict whether the modal frequency exceeding the natural frequency [6]. If the modal frequency exceeds the natural frequency the bone is going to fail [7]. Many studies are conducted to predict the modal frequencies of the femur bone, but only a few can develop/model the proximal femur bone for the analysis purpose [8-9].

\footnotetext{
Revised Manuscript Received on April 25, 2019. India

Vaishali Chaudhry, Graphic Era Deemed to be University, Dehradun,

Anubhav Kumar, Graphic Era Deemed to be University, Dehradun, India

Shilpa N, Department of Medical Electronics, MVJ College of Engineering, Bangalore, India

Shwetank Avikal, Graphic Era Hill University, Dehradun, India

Nithin Kumar K.C., Graphic Era Deemed to be University, Dehradun, India
}

In the design of femur bone implants stress analysis and modal analysis plays an important role and finding the critical places where the fracture occurs will help us to design the implants properly [10].

In this work, we are carrying out the modal analysis of the femur bone for three possible bone implant materials for given boundary conditions. Such that the implant material is selected and modal frequencies are studied.

\section{MATERIALS AND METHOD}

Following heads, materials, and methods followed in this work are discussed.

\subsection{Materials}

Natural bone, AZ31, and Stainless steel 316L are selected in this study. These materials are generally used in the manufacturing of bone implants. The mechanical properties of natural bone are Young's modulus 3-20GPa, Poisson's ratio 0.33 , and density $1.8-2.1 \mathrm{~g} / \mathrm{cm}^{3}$.

The mechanical properties of AZ31 Young's modulus are $45 \mathrm{GPa}$, Poisson's ratio 0.35 and Density $1.81 \mathrm{~g} / \mathrm{cm}^{3}$, and For Stainless steel 316L are Young's modulus is 200GPa, Poisson's ratio 0.30 and Density $7.8 \mathrm{~g} / \mathrm{cm}^{3}[11-12]$.

\subsection{Methodology}

Fig. 1 shows the method followed in this study. 


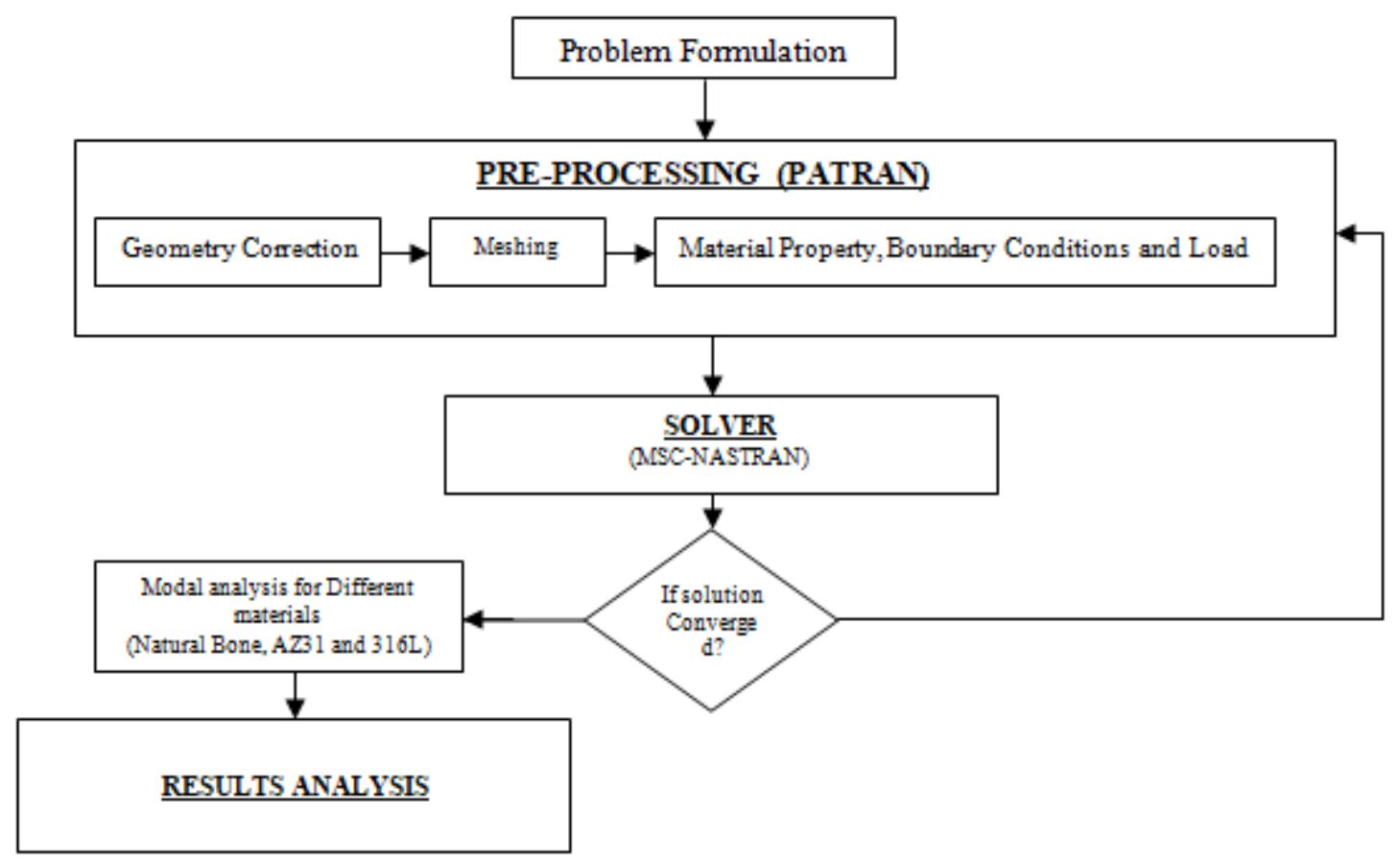

Fig. 1. Methodology

\subsection{Modeling and Meshing}

The CT-scan grayscale data is used to model femur bone using ITK-SNAp open-source software. The Threedimensional model is preprocessed using MSC-Patran and we selected CQUID 4 element and it gives accurate results in comparison with the other elements. The element size is decided after applying the convergence criteria. The element size for this study is $2 \mathrm{~mm}$.

\subsection{Boundary Conditions}

The boundary condition selected from the literature. The daily activity such as walking is used in our study [12]. The

femur head is fixed and $750 \mathrm{~N}$ load is applied at the Knee joint.

\section{RESULTS}

Modal analysis for natural bone, AZ31, and Steel 316L are carried out by meeting all the boundary conditions for femur bone. The results are discussed below.

\subsection{Modal frequencies for natural femur bone}

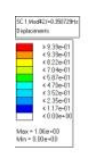

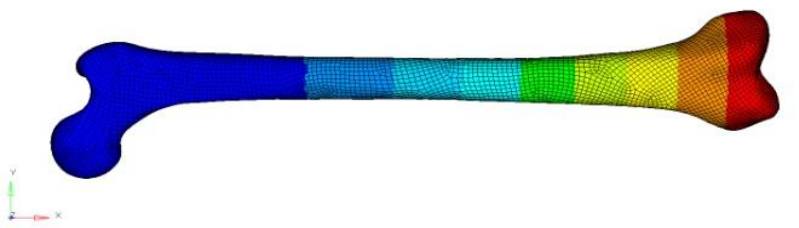

Fig. 2 Mode 1

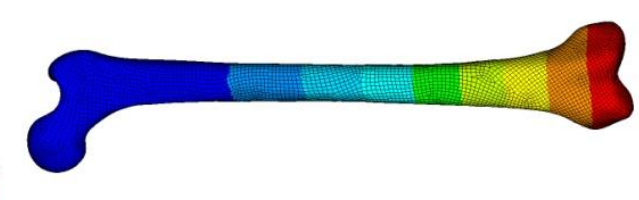

Fig. 3 Mode 2

Fig. 2 and Fig. 3 shows the modal frequencies for Mode 1 and Mode 2 for natural bone materials are $0.328 H z$ and $0.350 \mathrm{~Hz}$.

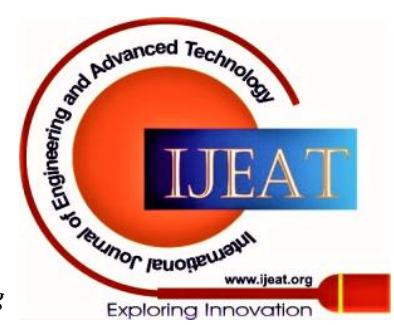




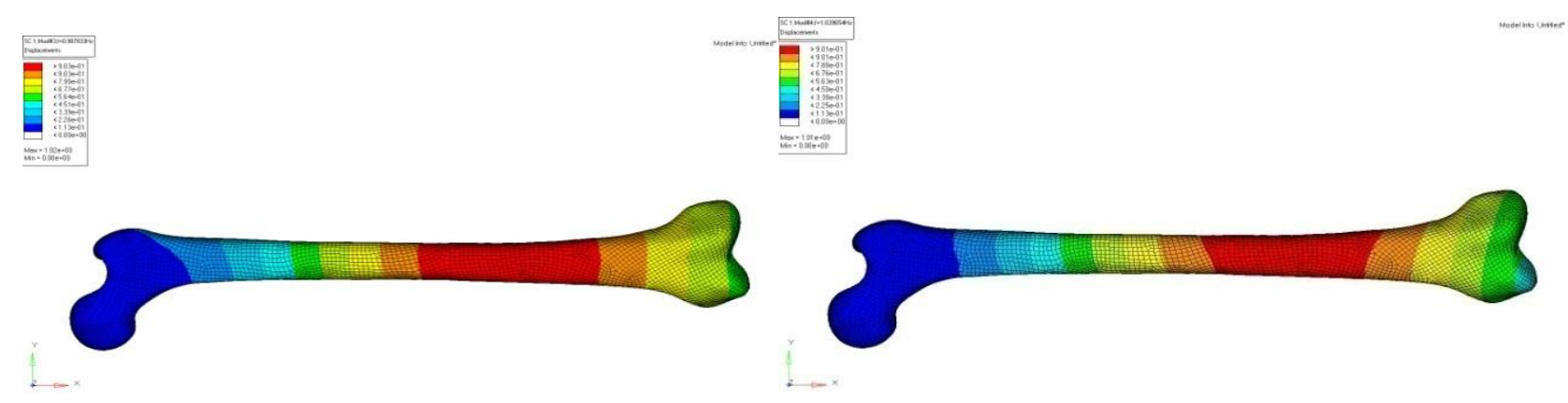

Fig. 4 Mode 3

Fig. 5 Mode 4

Fig.4 and Fig. 5 shows the modal frequencies for Mode 3 and Mode 4 for natural bone materials are $0.988 H z$ and $1.039 H z$.

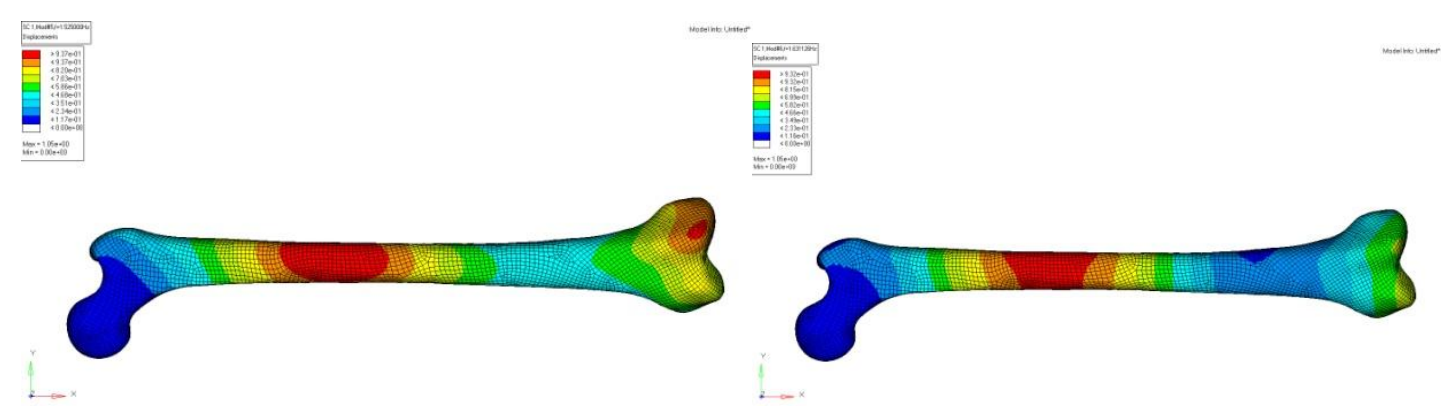

Fig. 6 Mode 5

Fig. 7 Mode 6

Fig.6 and Fig. 7 show the modal frequencies for Mode 5 and Mode 6 for natural bone materials are $1.524 \mathrm{~Hz}$ and $1.631 \mathrm{~Hz}$.

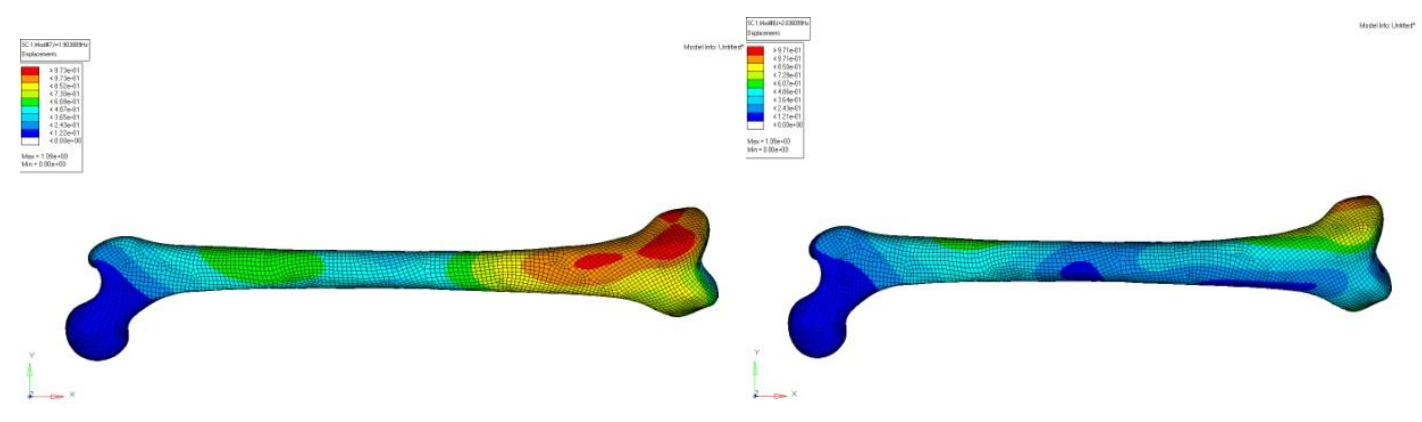

Fig. 8 Mode 7

Fig. 9 Mode 8

Fig.8 and Fig. 9 show the modal frequencies for Mode 7 and Mode 8 for natural bone materials of Femur bone are $1.903 \mathrm{~Hz}$ and $2.036 \mathrm{~Hz}$. 


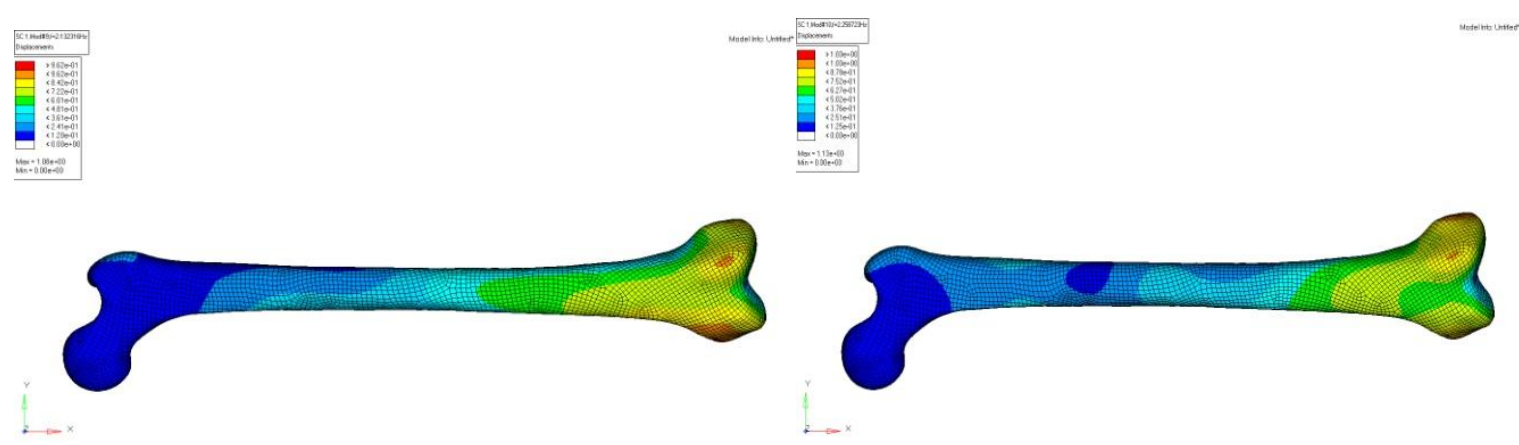

Fig. 10 Mode 9

Fig. 11 Mode 10

Fig.10 and Fig. 11 show the modal frequencies for Mode 9 and Mode 10 for natural bone materials of the Femur bone are $2.136 \mathrm{~Hz}$ and $2.258 \mathrm{~Hz}$.

Similarly, the modal analysis for AZ31 and Stainless steel $316 \mathrm{~L}$ is carried out.

Table 1 Modal frequencies

\begin{tabular}{cccc}
\hline \multirow{2}{*}{ Modes/ Material } & \multicolumn{3}{c}{ Modal frequency (Hz) } \\
\cline { 2 - 4 } & Natural bone & AZ31 & Stainless Steel 316L \\
\hline 1 & 0.328 & 1.502 & 3.120 \\
\hline 2 & 0.350 & 1.606 & 3.338 \\
\hline 3 & 0.988 & 4.530 & 9.403 \\
\hline 4 & 1.039 & 4.759 & 9.896 \\
\hline 5 & 1.524 & 6.981 & 14.516 \\
\hline 6 & 1.631 & 7.459 & 15.526 \\
\hline 7 & 1.903 & 8.395 & 18.123 \\
\hline 8 & 2.036 & 9.286 & 19.381 \\
\hline 9 & 2.132 & 9.690 & 20.297 \\
\hline 10 & 2.258 & 10.292 & 21.150 \\
\hline
\end{tabular}
natural bone modal frequency varies from $0.328 \mathrm{~Hz}$ to $2.258 \mathrm{~Hz}$ for different modes. The modal frequency of AZ31 varies from $1.502 \mathrm{~Hz}$ to $10.292 \mathrm{~Hz}$ and whereas $3.120 \mathrm{~Hz}$ to $21.150 \mathrm{~Hz}$ modal frequency for $316 \mathrm{~L}$. These modal frequencies are minimal as compared to the natural frequency of the natural bone. If the fracture occurs than it occurs in the femoral shaft.

\section{CONCLUSIONS}

Modal analysis of femur bone is carried out meeting all the boundary conditions. The following conclusions were drawn from this study.

$>$ The modal frequencies for Natural Femur bone vary from $0.328 \mathrm{~Hz}$ to $2.258 \mathrm{~Hz}$ for Mode 1 to Mode 10 .

$>$ The modal frequencies for AZ31 vary from $1.502 \mathrm{~Hz}$ to $10.292 \mathrm{~Hz}$ for Mode1 to Mode 10.

$>$ The modal frequencies for $316 \mathrm{~L}$ vary from $3.120 \mathrm{~Hz}$ to $21.150 \mathrm{~Hz}$ for Mode1 to Mode 10.

$>$ These frequencies must be minimal as compared to the natural frequency of the Femur bone.

$>$ AZ31 is best suited for the fabrication of bone implants because of its lightweight in comparison with $316 \mathrm{~L}$ material. Also, this is biodegradable in the human body over the period.
The results are shown in Table 1. It is observed that for

\section{REFERENCES}

1. Majid Mirzaei, et. al., Analysis of Strength And Failure Pattern Of Human Proximal Femur Using Quantitative Computed Tomography (Qct)-Based Finite Element Method, Bone 64 (2014) 108-114.

2. Bonel. N, et. al., Effect of Amputation Level on The Stress Transferred to The Femur by An artificial Limb Directly Attached to The Bone, Medical Engineering and Physics 35 (2013) 1744- 1753.

3. Sandeep Kumar Parashara, Jai Kumar Sharma, A Review on Application of Finite Element Modeling in Bone Biomechanics, Icems-2016,1-5.

4. Richard R. Pelkersection, Subrata Saha, Stress Wave Propagation in Bone, J. Biomechanics Vol. 10 No. 7, 1983, Pp 481-489.

5. A.T.M. Phillips, The Femur As A Musculo-Skeletal Construct: A Free Boundary Condition Modelling Approach, Medical Engineering \& Physics 31 (2009) 673-680.

6. Uday v. Pise, et. al., A B-Spline Based Heterogeneous Modeling and Analysis of Proximal Femur with Graded Element, Journal Of Biomechanics, 42(2009)1981-1988.

7. Yuichi Watanabe, et. al., Biomechanical Study of the Resurfacing Hip Arthroplasty: Finite Element Analysis Of The Femoral Component, The Journal Of Arthroplasty Vol. 15 No. 4 June 2000, 505-511.

8. R. Shahar, et. al., Stress And Strain Distribution in the Intact Canine Femur: Finite Element Analysis, Medical Engineering \& Physics 25 (2003) 387-395.

9. Edward G. Sutter, et. al., A Biomechanical Evaluation Of Femoroplasty Under Simulated Fall Conditions, J Orthop Trauma 2010;24:95-99.

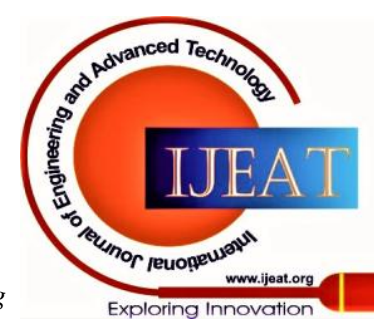


10. Nir Trabelsi, et. al., Patient-Specific Finite Element Analysis of the Human Femur-A Double-Blinded Biomechanical Validation, Journal Of Biomechanics 44(2011) 1666-1672.

11. C.J. Wang, et. al., Finite Element Analysis of A Gamma Nail within A Fractured Femur, Medical Engineering \& Physics 20 (1998) 677683.

12. Nithin Kumar KC, et. al., Biomechanical stress analysis of human femur bone using ANSYS, Materials Today: Proceedings 2 (2015) $2115-2120$ 